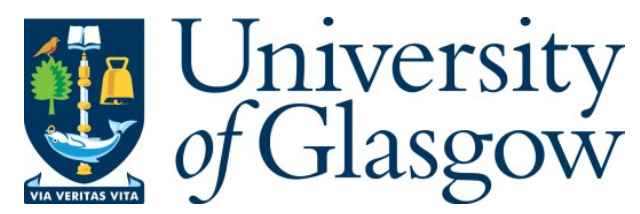

Gibson, G.M., Dienerowitz, M., Kelleher, P.A., Harvey, A.R., and Padgett, M.J. (2013) A multi-object spectral imaging instrument. Journal of Optics, 15 (8). 085302. ISSN 2040-8978

Copyright (C 2013 The Authors

http://eprints.gla.ac.uk/84110

Deposited on: 08 May 2014

Enlighten - Research publications by members of the University of Glasgow http://eprints.gla.ac.uk 


\title{
A multi-object spectral imaging instrument
}

\author{
G M Gibson, M Dienerowitz, P A Kelleher, A R Harvey and M J Padgett
}

School of Physics and Astronomy, SUPA, University of Glasgow, G12 8QQ, UK

E-mail: Graham.Gibson@glasgow.ac.uk

Received 17 May 2013, accepted for publication 12 July 2013

Published 30 July 2013

Online at stacks.iop.org/JOpt/15/085302

\begin{abstract}
We have developed a snapshot spectral imaging system which fits onto the side camera port of a commercial inverted microscope. The system provides spectra, in real time, from multiple points randomly selected on the microscope image. Light from the selected points in the sample is directed from the side port imaging arm using a digital micromirror device to a spectrometer arm based on a dispersing prism and CCD camera. A multi-line laser source is used to calibrate the pixel positions on the CCD for wavelength. A CMOS camera on the front port of the microscope allows the full image of the sample to be displayed and can also be used for particle tracking, providing spectra of multiple particles moving in the sample. We demonstrate the system by recording the spectra of multiple fluorescent beads in aqueous solution and from multiple points along a microscope sample channel containing a mixture of red and blue dye.
\end{abstract}

Keywords: spectral imaging, multi-object spectrometer, digital micromirror device

S Online supplementary data available from stacks.iop.org/JOpt/15/085302/mmedia

(Some figures may appear in colour only in the online journal)

\section{Introduction}

Imaging spectrometers are used to acquire spectrally resolved images of an object or scene. They typically record data over three dimensions; two spatial and one spectral, where the complete $(x, y, \lambda)$ data set is commonly referred to as a datacube consisting of individual elements called voxels [1]. In order to build the datacube, imaging spectrometers traditionally require some form of timesequential scanning [2]. A number of technologies have been developed including, whiskbroom and pushbroom where a 1 or 2D detector array is used to scan a column or slice of the datacube [3], a filtered camera to record slices scanned along the spectral dimension [4] and Fourier-transform imaging spectrometry [5-7]. Snapshot imaging spectrometers, on the other hand, can record the entire datacube without the need for scanning and have the advantage of improved light-collection efficiency [8].

There exists a number of snapshot techniques for spectral imaging [9-12]. They have applications in astronomy and microscopy [13] and also in opthalmology [14, 15].
Snapshot imaging spectrometers generally require a trade-off between spatial and spectral resolution and hence the spectral resolution is normally limited. Also, for high resolution, real time, spectral imaging the required data transfer rate from the detector array, and the processing of large datacubes presents a significant challenge.

Here we report the development of an imaging spectrometer which is based on a digital micromirror device (DMD) and is interfaced to a commercial inverted microscope. The DMD is used to divert subsets of the microscope image, corresponding to multiple points of interest selected from the full field-of-view, to a spectrometer. DMDs, consisting of hundreds of thousands of individually addressable moving micromirrors, were originally developed for the display industry [16] but have also found applications in other areas including wavelength multiplexing [17]. They offer a method of modulating light which is fast, highly efficient and works over a broad range of wavelengths. A binary image displayed on the DMD allows patterns of micromirrors to control the reflection path of light in an optical system, each individual micromirror being 


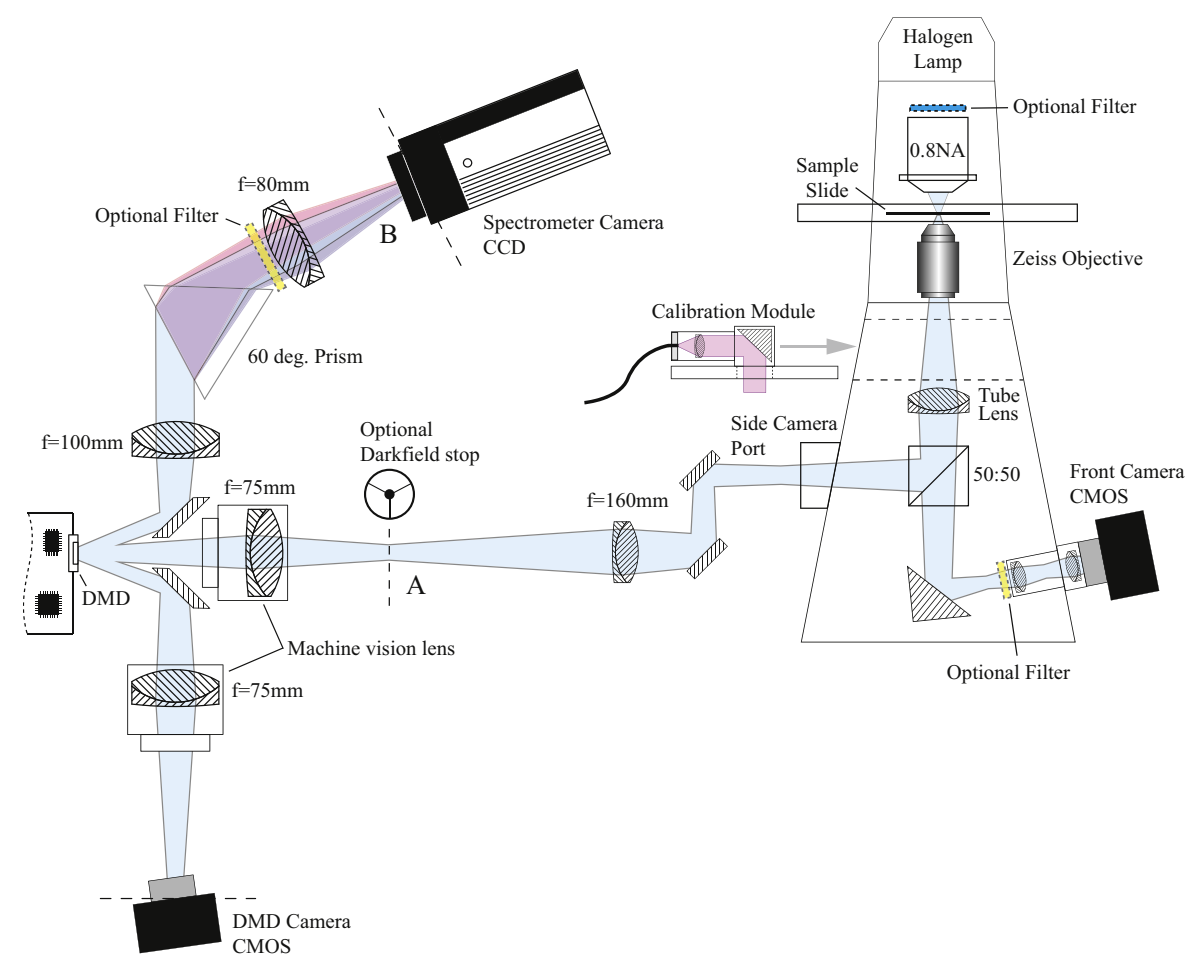

Figure 1. Schematic of the optical system. The system is based around a commercial inverted microscope (Zeiss Axiovert 200). Incorporated within the microscope is a 50:50 beamsplitter which splits the image between the front and side camera ports of the microscope. A CMOS camera provides both a live view of the sample and can be used for particle tracking. The image at the side port of the microscope is imaged onto a DMD chip which is used to send user-defined segments of the image to a spectrometer. The spectrometer consists of a $60^{\circ}$ prism and a cooled CCD camera. Optional filters allow the system to be used for fluorescence or darkfield [23] imaging. A and $\mathrm{B}$ are measurement points used to determine the optical throughput of the system.

switchable by $\pm 12^{\circ}$. The use of DMDs has been demonstrated in multi-object spectroscopy [18] and also applied to astronomical observations [19]. They have also been used to acquire optically sectioned images in fluorescence microscopy [20], improve optical sectioning in fiber-optic confocal microscopy [21] and enhance the dynamic range of digital optical microscope images [22]. Here the DMD deflects the user-defined regions of the microscope image from the imaging arm to the spectrometer arm of the system. The spectrometer arm is based on a dispersing prism and monochrome CCD camera. A calibration scheme is employed to calibrate the camera pixel positions for wavelength.

Control software allows the user to interactively select points of interest from within the full field-of-view of the microscope, providing spectral information from those points in real time. In addition, the DMD is capable of being updated at video frame rates which provides the opportunity to track moving objects while displaying their spectra. As demonstrations we track multiple fluorescent microbeads and show their evolving spectra whilst moving within the sample and show the time evolved spectra of red and blue dye mixing and flowing along a microchannel.

\section{System configuration}

A schematic of the optical system is shown in figure 1. The system is attached to a commercial inverted microscope
(Zeiss, Axiovert 200) compatible with a range of different objective lenses. To demonstrate the system at different magnifications and numerical apertures, two objective lenses, $100 \times 1.3$ NA (Zeiss, Plan-Neofluar) and $10 \times 0.25$ NA (Zeiss, EC Epiplan-Neofluar) were used. A $100 \mathrm{~W}$ halogen lamp with a $0.8 \mathrm{NA}$ condenser provides illumination of the sample. Incorporated within the microscope is a 50:50 beamsplitter cube which splits the image between the front and side camera ports of the microscope. A color CMOS camera (Mantra, G-046C) mounted on the front port of the microscope provides both a live view of the sample and can be used for particle tracking. The image at the side port of the microscope is imaged onto a DMD (Texas Instruments, LightCrafter with 0.3WVGA chipset) using a machine-vision lens (Qioptiq, Apo-Rodagon-D $2 \times$ ). The LED illumination and projection optics have been removed from the LightCrafter to allow direct access to the DMD chip, consisting of 608 pixels $\times$ 684 pixels of pitch $=7.6 \mu \mathrm{m}$, arranged in a diamond pattern geometry. Normally the image is reflected into an imaging arm which consists of a second identical machine-vision lens and a color CMOS camera (Prosilica, GC660C). Controlling the pattern displayed on the DMD, connected to a PC using a mini HDMI connection, allows the user to specify regions of the image to be directed from the imaging arm into a spectrometer arm consisting of a $60^{\circ}$ dispersing prism and a cooled CCD camera (QImaging, Retiga Exi), 1392 pixels $\times$ 1040 pixels. The remainder of the image can be used to check the alignment of the system by matching the missing regions 


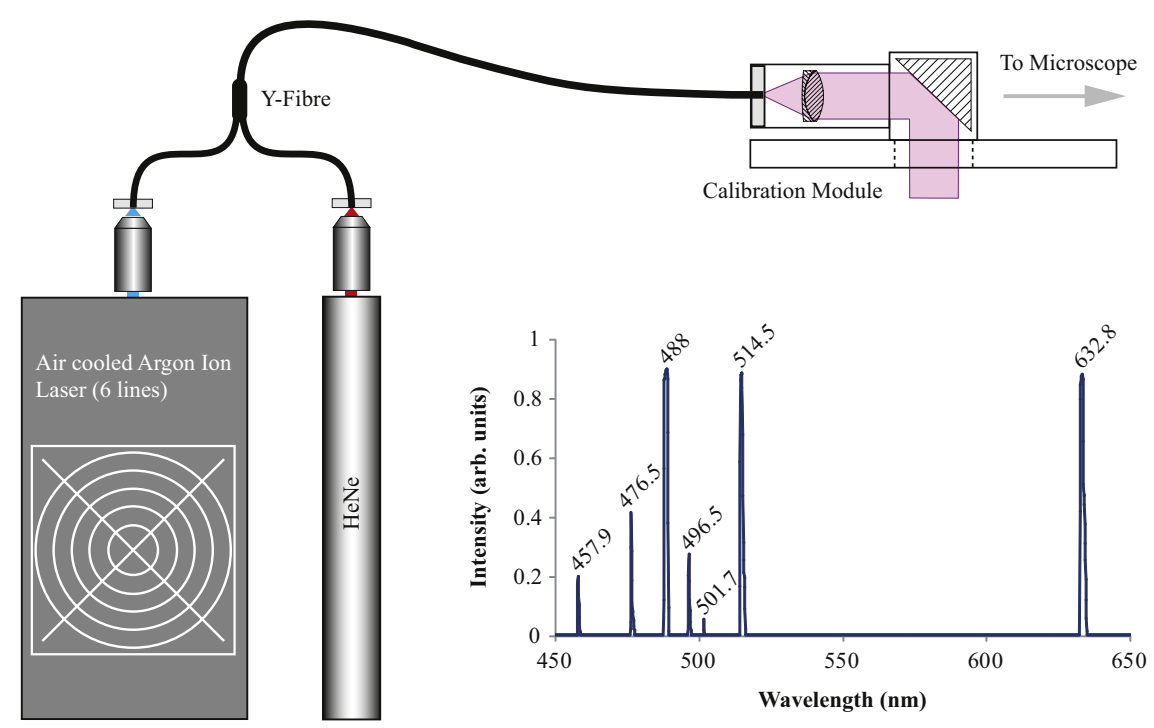

Figure 2. Schematic of the calibration optical system. The laser beams from a multi-wavelength argon ion laser, consisting of six lines, and a helium neon laser are focused into a Y junction optical fiber which combines both sources at the fiber output. The fiber is attached to a module which collimates the output from the fiber and can be inserted into the microscope, above the tube lens, to allow the system to be calibrated. Also shown is the measurement of the calibration source from a USB fiber coupled spectrometer with the peak wavelengths identified.

to the corresponding regions defined by the user in the full microscope image. A custom module allows a multi-line laser source to be easily coupled into the system for calibration. The calibration module can be inserted into the microscope directly above the tube lens and allows the pixel positions in the spectrometer camera to be calibrated for wavelength, as a function of the pixels selected in the image.

The multi-line laser calibration system is shown in figure 2. A multi-line air-cooled argon ion laser (Laser Graphics, 151 LGA) and a helium neon laser are combined using a 2 m length ' $Y$ ' splitter optical fiber assembly (Ocean Optics, $600 \mu \mathrm{m}$ core), providing a total of seven laser lines at the fiber output. The fiber is attached to a module which incorporates a collimating lens and the module can be inserted into the microscope, above the tube lens, to allow the system to be calibrated. A plot of the known calibration lines is shown in figure 2, measured using a USB fiber coupled spectrometer (Thorlabs, CCS100).

\section{Software}

The control software including the user interface was written in LabVIEW (National Instruments). The user can select multiple points of interest on the sample image simply by clicking the mouse pointer. A binary image is created on which a slit is drawn overlaying each point of interest. The dimensions of the slits can also be defined by the user and determines the resolution of the spectrometer. The image is then displayed on the DMD which controls the path of light to the spectrometer arm. The positions of the slits displayed on the DMD are used to extract the corresponding regions from the spectrometer CCD image showing the slits after dispersion from the $60^{\circ}$ prism. Image processing each of the extracted regions, averaging over the pixel columns and calibrating the wavelength scale, provides the spectra corresponding to each of the original user selected points of interest.

Particle tracking can be implemented on the image from the full field camera mounted on the front port of the microscope. Pattern-matching software, developed using the Vision Toolkit (National Instruments), allows multiple objects, and the corresponding positions of the slits, to be tracked. The DMD can be updated at video frame rates and hence allows the spectrometer slits to be positioned to correspond to the tracked objects, providing spectra of multiple tracked objects in real time.

\section{Calibration}

The positions of the pixels on the spectrometer CCD camera need to be calibrated for wavelength, which is also dependent upon the positions of the slits on the DMD. Inserting the calibration module into the microscope results in a uniform illumination of a large region of the field-of-view. Normally three points are selected, distributed from top to bottom and left to right, covering most of the field-of-view of the microscope. Changing the slit size allows a trade-off between resolution and optical throughput. A common slit size of 5 pixels wide $\times 25$ pixels high was used in the examples presented in this paper, resulting in a resolution better than $2.5 \mathrm{~nm}$. The second imaging arm is used to check the alignment of the points of interest to the slits displayed on the DMD chip. Scaling parameters are used to fit the wavelength scale to the spectra obtained from the spectrometer CCD image. A combination of offset and scaling factors determines the starting value and range of the wavelength scale, adjusted such that the shortest and longest wavelength peaks from the acquired spectra match the corresponding peaks from the calibration source, identified using the USB spectrometer. 

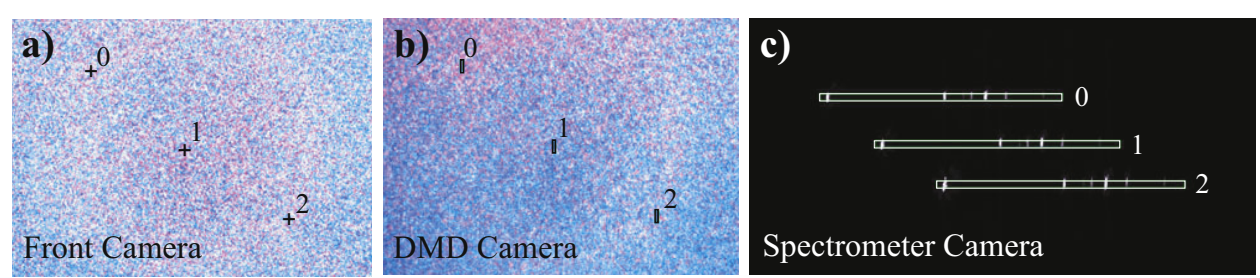

d)

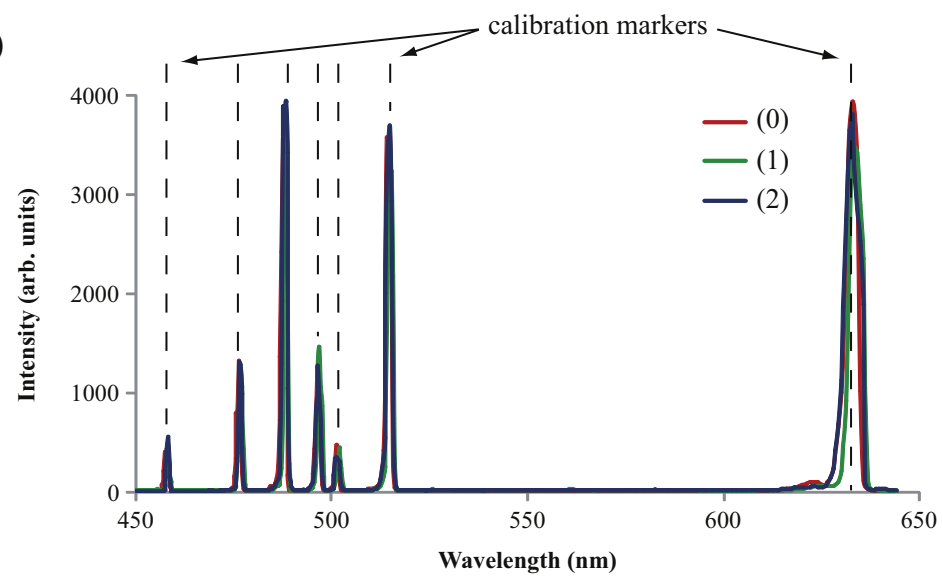

Figure 3. Results of the system calibration. (a) The calibration source fills a large area of the field-of-view from which multiple points of interest can be selected, shown as numbered crosses on the image. (b) Each point is used to define spectrometer slits which are displayed on the DMD. A CMOS camera displays the image reflected from the DMD minus the parts directed to the spectrometer arm. (c) Extended regions are extracted from the spectrometer camera image corresponding to the dispersed image of each slit. (d) Averaging columns of pixels provides the spectra and scaling parameters are used to fit the wavelength scale to the calibration markers from the known laser lines.

a)

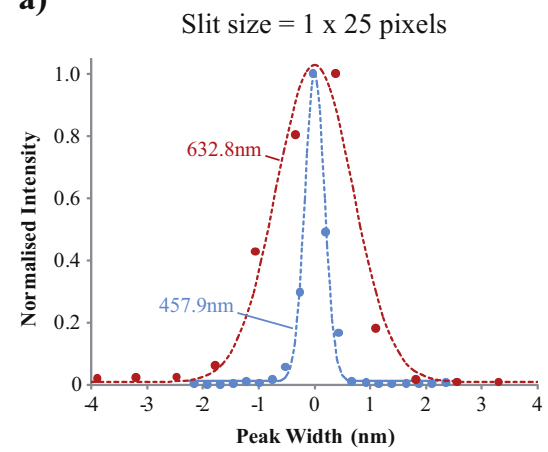

b)

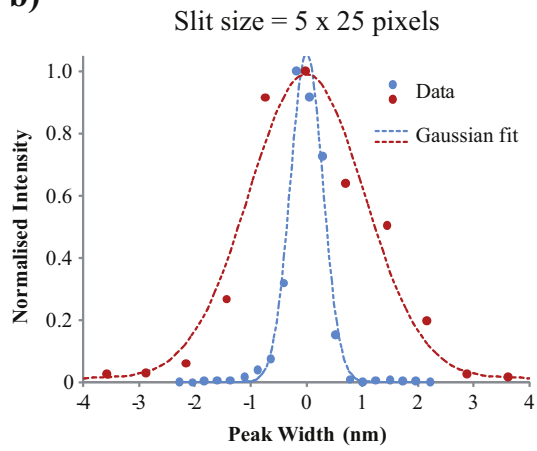

Figure 4. Measured peak width of the 457.9 and $632.8 \mathrm{~nm}$ lines for two different slit widths. (a) Narrowest possible slit width $=1$ pixel results in a FWHM $632.8 \mathrm{~nm}=1.63 \mathrm{~nm}$ and FWHM $_{457.9 \mathrm{~nm}}=0.42 \mathrm{~nm}$. (b) Slit width $=5$ pixels results in FWHM $632.8 \mathrm{~nm}=2.44 \mathrm{~nm}$ and $\mathrm{FWHM}_{457.9 \mathrm{~nm}}=0.69 \mathrm{~nm}$.

Due to the slightly nonlinear dispersion of the prism, an additional correction term is required in order to align the intermediate peaks. Figure 3 shows the results of a system calibration.

An important consideration is the resolution of the system when selecting the narrowest possible slit, in this case 1 pixel width on the DMD $(=10.8 \mu \mathrm{m})$. Each extracted region from the $\mathrm{CCD}$ readout measures 500 pixels $\times 100$ pixels (of size $6.45 \mu \mathrm{m}$ ) and corresponds to a wavelength range of 450-650 $\mathrm{nm}$. The estimated average wavelength dispersion (AWD) is calculated to be $62 \mathrm{~nm} \mathrm{~mm}{ }^{-1}$. Figure 4 shows experimental measurements of the FWHM of the $457.9 \mathrm{~nm}$ and $632.8 \mathrm{~nm}$ lines for slit sizes of 1 pixels $\times 25$ pixels and 5 pixels $\times 25$ pixels respectively. The beam width on the prism face was measured to be approximately $10 \mathrm{~mm}$, which corresponds to a calculated diffraction-limited FWHM of $0.23 \mathrm{~nm}$ and $0.92 \mathrm{~nm}$ for the blue and red lines respectively. It is important to note that due to the diagonal orientation of the micromirrors on the DMD, the effective width of the narrowest slit is ill defined.

The optical throughput of the system depends on various factors including the reflectivity of the individual micromirrors, the fill factor and transmission and reflective losses at the window of the DMD device. Also, different options for the microscope beamsplitter are possible, controlling the splitting ratio between the front and side camera ports. Measurement points A and B, shown in figure 1, are convenient locations for measuring the optical throughput 

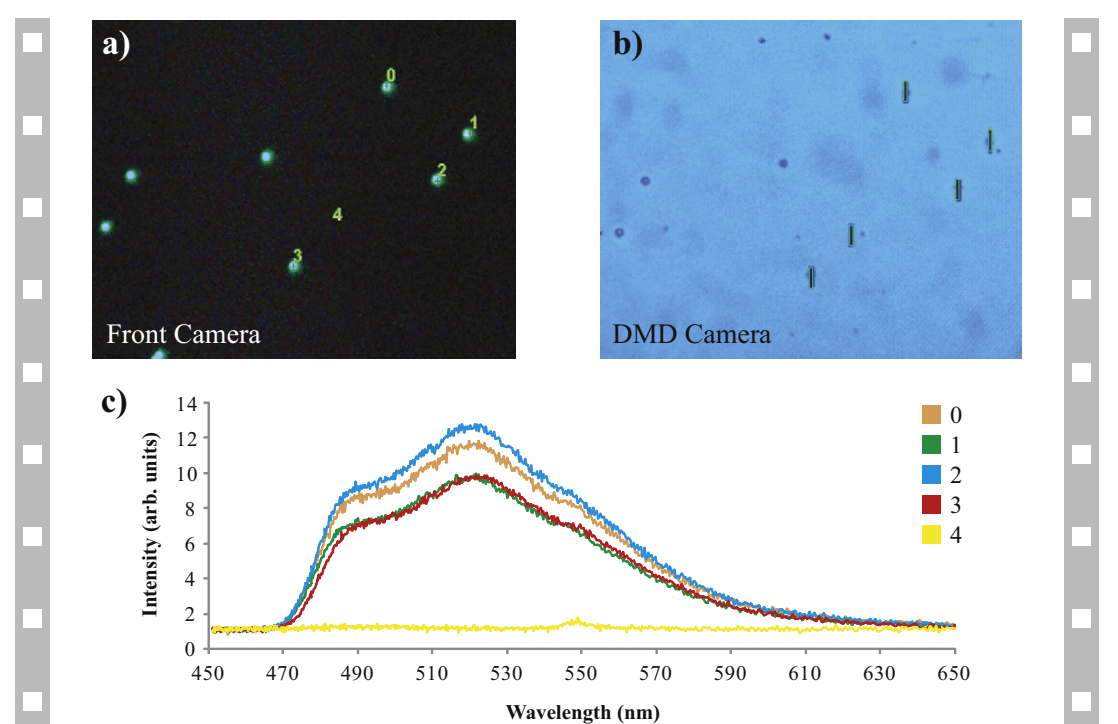

Figure 5. Results of tracking $1 \mu \mathrm{m}$ fluorescent beads. (a) The front camera image showing the fluorescent beads and five selected points of interest. (b) The DMD camera image showing the five corresponding spectrometer slits. (c) The spectra corresponding to the selected points of interest. See accompanying movie (available at stacks.iop.org/JOpt/15/085302/mmedia), Tracking fluorescent beads.mov (12 MB).

of the spectrometer instrument. Using the HeNe laser as the illumination, and pointing all the mirrors on the DMD towards the spectrometer arm, we measure an optical throughput of $35 \%$.

\section{Example applications}

To demonstrate the system we show two applications. The first of these shows the spectra of multiple fluorescent beads moving in a sample. An aqueous sample containing $1 \mu \mathrm{m}$ diameter fluorescent beads (Polysciences, inc., Fluoresbrite ${ }^{\circledR}$ YG Microspheres, cat. no. 17154) was prepared on a microscope slide, fixed with a coverglass, and mounted in the sample holder of the microscope stage. A $100 \times$, 1.3NA objective lens was used to image the sample, giving a field-of-view of $101 \mu \mathrm{m} \times 75 \mu \mathrm{m}$. An interference filter (440, $10 \mathrm{~nm}$ bandwidth) located above the illumination condenser selects the excitation wavelength band for the bead sample. Corresponding emission filters (475 nm, long pass), placed before the front camera and spectrometer camera, block the excitation wavelengths while passing the emission wavelengths. Using separate emission filters allows the DMD camera to see the unfiltered light and to record the slit positions as they are updated on the DMD. Figure 5 represents a movie (available at stacks.iop.org/JOpt/15/085302/mmedia) showing the beads being tracked and their corresponding spectra shown. The movie shows the spectra from five points of interest corresponding to three beads exhibiting Brownian motion, one bead stuck to the coverglass and one background measurement. The integration time of the spectrometer camera was set to $100 \mathrm{~ms}$ and the resulting spectra smoothed using a recursive filter with an $\alpha$ constant of 0.05 . In addition to the spectra, the movie shows the slit pattern on the DMD being updated to coincide with the positions of the tracked beads. Here we record the movie at ten frames per second. This is limited by the rate at which images can be acquired from the cooled CCD spectrometer camera.

The second application shows the time evolving spectra at multiple locations distributed along a sample channel. A sample channel was prepared by fixing two adjacent microscope coverglasses, using UV curing glue, to a microscope slide and capped using a third coverglass. The channel was initially filled with a red dye consisting of a 1:1 dilution of red food coloring with distilled water. A similar blue dye was prepared and a drop was placed at one end of the channel and allowed to flow into and mix with the red dye. The halogen lamp and condenser, as described in section 2, provides the illumination. Here a $10 \times$, $0.25 \mathrm{NA}$ objective lens was used to image the sample, giving a field-of-view of $1030 \mu \mathrm{m} \times 766 \mu \mathrm{m}$. The integration time of the spectrometer camera was set to $10 \mathrm{~ms}$. Figure 6 shows the time evolved spectra of the blue dye mixing with, and eventually replacing, the red dye in the channel. Here we are taking full advantage of the fact that measurements can be taken at multiple locations along the vertical (non-dispersing) axis without restrictions due to spectral overlap.

\section{Conclusions}

We have developed a multi-object snapshot spectral imaging system where the spectra from randomly accessed points in a microscope image can be acquired in real time. The spectrometer operates in slit mode by defining a slit for each source in the image, displayed on a DMD. The system can be used for brightfield and fluorescence imaging and is also compatible with darkfield imaging by inserting a center stop at location A in figure 1 [23]. We have used particle tracking software to track the positions of multiple fluorescent microbeads in aqueous solution and updated the DMD to obtain spectra of the beads as they moved in the sample. Such an approach has potential applications for in vivo samples 

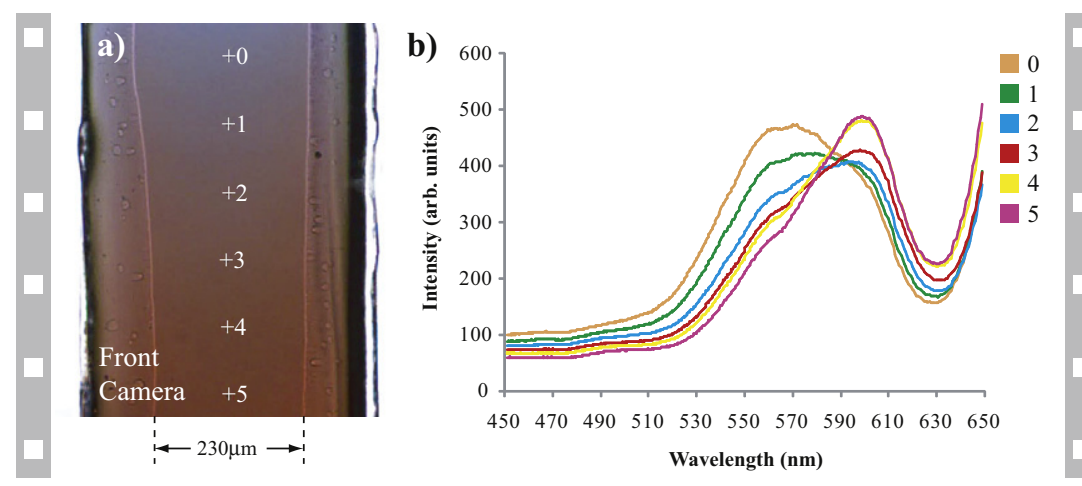

Figure 6. Results of red and blue dye mixing and flowing in a channel. (a) The front camera showing the brightfield image of the sample and six selected points of interest. (b) The spectra corresponding to the selected points of interest. See accompanying movie (available at stacks.iop.org/JOpt/15/085302/mmedia), Red and blue dye in microchannel.mov (2.2 MB).

where there is movement of the sample due to respiration. We acquired spectra from multiple positions along a microscope sample channel in which two colors of dye mixed and flowed, showing the time evolved spectra. An important consideration is the possibility of spectra overlapping from different sources aligned along the dispersion axis. While this was easily avoided in the examples shown here it may be a limitation for samples having closely spaced sources. A possible solution to spectral overlap is the implementation of a spectral unmixing algorithm [24].

\section{Acknowledgments}

MJP acknowledges support from the Royal Society and EPSRC. ARH acknowledges support from EPSRC.

\section{References}

[1] Descour M and Dereniak E 1995 Computed-tomography imaging spectrometer: experimental calibration and reconstruction results Appl. Opt. 34 4817-26

[2] Harvey A R, Beale J, Greenaway A H, Hanlon T J and Williams J 2000 Technology options for imaging spectrometry Proc. SPIE 4132 13-24

[3] Green R O et al 1998 Imaging spectroscopy and the airborne visible/infrared imaging spectrometer (AVIRIS) Remote Sens. Environ. $65227-48$

[4] Gat N 2000 Imaging spectroscopy using tunable filters: a review Proc. SPIE 4056 50-64

[5] Barducci A, Guzzi D, Lastri C, Marcoionni P, Nardino V and Pippi I 2010 Theoretical aspects of Fourier transform spectrometry and common path triangular interferometers Opt. Express 18 11622-49

[6] Schumann L W and Lomheim T S 2002 Infrared hyperspectral imaging Fourier transform and dispersive spectrometers: comparison of signal-to-noise based performance Proc. SPIE 4480 1-14

[7] Harvey A R and Fletcher-Holmes D W 2004 Birefringent Fourier-transform imaging spectrometer Opt. Express 12 5368-74

[8] Hagen N, Kester R T, Gao L and Tkaczyk T S 2012 Snapshot advantage: a review of the light collection improvement for parallel high-dimensional measurement systems Opt. Eng. 51111702

[9] Johnson W R, Wilson D W and Bearman G 2006 Spatial-spectral modulating snapshot hyperspectral imager Appl. Opt. 45 1898-908
[10] Gorman A, Fletcher-Holmes D W and Harvey A R 2010 Generalization of the Lyot filter and its application to snapshot spectral imaging Opt. Express 18 5602-8

[11] Fletcher-Holmes D W and Harvey A R 2005 Real-time imaging with a hyperspectral fovea J. Opt. A: Pure Appl. Opt. 7 S298-302

[12] Gao L, Kester R T and Tkaczyk T S 2009 Compact image slicing spectrometer (ISS) for hyperspectral fluorescence microscopy Opt. Express 17 12293-308

[13] Gao L, Kester R T, Hagen N and Tkaczyk T S 2010 Snapshot image mapping spectrometer (IMS) with high sampling density for hyperspectral microscopy Opt. Express 18 14330-44

[14] Johnson W R, Wilson D W, Fink W, Humayun M and Bearman G 2007 Snapshot hyperspectral imaging in ophthalmology J. Biomed. Opt. 12014036

[15] Mordant D J, Al-Abboud I, Muyo G, Gorman A, Sallam A, Ritchie P, Harvey A R and McNaught A I 2011 Spectral imaging of the retina Eye 25 309-20

[16] Sampsell J B 1994 Digital micromirror device and its application to projection displays J. Vac. Sci. Technol. B 12 3242-6

[17] McConnell G, Poland S and Girkin J M 2006 Fast wavelength multiplexing of a white-light supercontinuum using a digital micromirror device for improved three-dimensional fluorescence microscopy Rev. Sci. Instrum. 77013702

[18] Voropai E S, Gulis I M, Kupreev A G, Kaplevskii K N, Kostyukevich A G, Radko A E and Shevchenko K A 2010 Multi-object spectrometer with micromirror array J. Appl. Spectrosc. 77 285-92

[19] Meyer R D, Kearney K J, Ninkov Z, Cotton C T, Hammond P and Statt B D 2004 RITMOS: a micromirror-based multi-object spectrometer Proc. SPIE 5492 200-19

[20] Fukano T and Miyawaki A 2003 Whole-field fluorescence microscope with digital micromirror device: imaging of biological samples Appl. Opt. 42 4119-24

[21] Lane P M, Dlugan A L P, Richards-Kortum R and MacAulay C E 2000 Fiber-optic confocal microscopy using a spatial light modulator Opt. Lett. 25 1780-2

[22] Adeyemi A A, Barakat N and Darcie T E 2009 Applications of digital micro-mirror devices to digital optical microscope dynamic range enhancement Opt. Express 17 1831-43

[23] Dienerowitz M, Gibson G, Dienerowitz F and Padgett M 2012 Expanding the toolbox for nanoparticle trapping and spectroscopy with holographic optical tweezers $J$. Opt. 14045003

[24] Grahn H F and Geladi P (ed) 2007 Techniques and Applications of Hyperspectral Image Analysis (New York: Wiley) 